\title{
A Canadian framework for managing prostate cancer during the COVID-19 pandemic: Recommendations from the Canadian Urologic Oncology Group and the Canadian Urological Association
}

Andrea Kokorovic, MD ${ }^{1}$; Alan I. So, MD²; Sebastien J. Hotte, MD³; Peter C. Black, MD²; Brita Danielson, $\mathrm{MD}^{4}$; Urban Emmenegger, $\mathrm{MD}^{5}$; Antonio Finelli, $\mathrm{MD}^{6}$; Tamim Niazi, $\mathrm{MD}^{7}$; Frederic Pouliot, $\mathrm{MD}^{8}$; Bobby Shayegan, $\mathrm{MD}^{9}$; Srikala Sridhar, $\mathrm{MD}^{10}$; Eric Vigneault, $\mathrm{MD}^{11}$; Andrew Loblaw, $\mathrm{MD}^{12}$; Ricardo Rendon, $\mathrm{MD}^{13}$

${ }^{1}$ The University of Texas MD Anderson Cancer Center, Houston, TX, United States; ${ }^{2}$ Department of Urologic Sciences, University of British Columbia, Vancouver, BC, Canada; ${ }^{3}$ Juravinski Cancer Centre, McMaster University, Hamilton, ON, Canada; ${ }^{4}$ Department of Oncology, Division of Radiation Oncology, University of Alberta, Edmonton, AB, Canada; ${ }^{5}$ Department of Medical Oncology, Sunnybrook Odette Cancer Centre, Toronto, ON, Canada; ${ }^{6}$ Princess Margaret Cancer Centre, University of Toronto, Toronto, ON, Canada; ${ }^{7}$ Department of Oncology, Division of Radiation Oncology, McGill University, Montreal QC, Canada; ${ }^{8}$ Division of Urology, Department of Surgery, Université Laval, Quebec City, QC, Canada; ${ }^{9}$ Division of Urology, McMaster University, Hamilton, ON, Canada; ${ }^{10}$ Division of Hematology and Medical Oncology, Princess Margaret Cancer Centre, University Health Network, University of Toronto, Toronto, ON, Canada; ${ }^{11}$ Department of Radiation Oncology, CHUQ, Université Laval, Quebec City, QC, Canada; ${ }^{12}$ Department of Radiation Oncology, Sunnybrook Odette Cancer Centre, Toronto, ON, Canada; ${ }^{13}$ Department of Urology, Dalhousie University, Halifax, NS, Canada

Cite as: Can Urol Assoc J 2020 April 28; Epub ahead of print. http://dx.doi.org/10.5489/cuaj.6667

Published online April 28, 2020

$* * *$

\section{Introduction}

The COVID-19 pandemic has had an unprecedented impact on all aspects of healthcare. One widespread strategy to mitigate the burden of disease is to limit nonessential exposure to healthcare settings by canceling office visits and non-emergent surgeries. The underlying concern is that there is an unknown proportion of patients and staff who are asymptomatic carriers and testing capacity is insufficient to test everyone ${ }^{1}$. The COVID-19 pandemic presents a unique challenge for oncology for several reasons. Patients with cancer might be more likely to get COVID-19 and have serious adverse outcomes, including intensive care admissions, ventilator requirements and death ${ }^{2,3}$. Furthermore, patients undergoing surgery, including select oncologic cases, may be at high risk for postoperative mortality ${ }^{4}$. In addition, intubation is a procedure that carries high risk of spreading the virus to members of the healthcare team present in the operating room. These complexities in cancer care have resulted in the release of several guidelines regarding management of oncology patients during the COVID-19 $\mathrm{era}^{5-9}$. Two recent Canadian Urological Association (CUA) publications outline recommendations on surgical 
triaging and the use of systemic therapies in genitourinary malignancies ${ }^{7,9}$. The purpose of this publication is to provide a multidisciplinary framework focused on prostate cancer management in the setting of the COVID-19 global pandemic within the Canadian context.

\section{General principles}

1. This consensus statement is a guide to help physicians manage prostate cancer during the acute phase of a pandemic. Treatment prioritization must take into account regional differences in infection rates, resource capacity and mitigation efforts. The current pandemic has had a widespread reach across all Canadian communities, but we recognize that the degree of impact varies, and that provincial and institutional policies are not uniform. Physicians must continue to monitor a continuously evolving situation and make adjustments to clinical decisions as deemed appropriate.

2. The risk of serious morbidity resulting from SARS-CoV-2 infection may outweigh the competing risk of prostate cancer in many men. Observation of prostate cancer in carefully selected patients does not increase long-term mortality ${ }^{10,11}$, and therefore shortterm treatment delays are unlikely to lead to disease progression and worse outcomes . All management decisions should be based on this core principle.

3. Appropriate patient counseling and shared decision making is strongly encouraged. Men diagnosed with prostate cancer have increased anxiety and psychological distress $^{12,13}$. This will undeniably become amplified in the setting of a global health crisis. Despite resource restrictions and changes in treatment recommendations, physicians must continue to address the needs of patients and involve them in the decision-making process. This approach may decrease patient anxiety levels and improve outcomes once regular practice resumes.

4. Prioritization must be given to limiting exposures of patients and healthcare workers to SARS-CoV-2. Implementation of telehealth visits significantly reduces the risk of infection among frontline personnel and patients, but also preserves critically needed hospital resources. For these reasons, telehealth visits are strongly encouraged. In-person consultations should be limited to men with new symptoms, those requiring a physical examination and for the evaluation and management of treatment-related serious adverse events. In men who require an in-person assessment, consideration should be given to not repeating visits when two specialists are consulted, nor when pre-operative assessment is needed. The healthcare provider should coordinate their needs to minimize patient's visits. 


\section{Screening and detection}

1. The CUA endorses prostate cancer screening and detection in appropriately selected $m^{14}$. However, the public health benefit from these recommendations is derived from long-term implementation and has no role in an acute setting. Therefore, we recommend cessation of routine prostate-specific antigen (PSA) screening in asymptomatic men until resolution of this pandemic.

2. In men with a suspicion of asymptomatic localized prostate cancer (based on PSA testing or clinical exam) we recommend delay of further investigations. This includes digital rectal examination (DRE), cross sectional or prostate imaging and transrectal ultrasound (TRUS) guided or perineal biopsies. These procedures increase patient and occupational exposure to SARS-CoV-2, utilize healthcare resources and are unlikely to improve patient outcomes in the short term. MRI has become a preferred imaging modality for diagnosis and staging of prostate cancer, however access is currently restricted and therefore its use should be limited for staging of high-risk cases when clinically indicated (see below). The risk of TRUS biopsy related sepsis is of particular concern given the potential severity of this complication, which can lead to hospitalization and further risk of exposure to the virus. A secondary concern is that of possible fecal SARS-CoV-2 transmission arising from the gastrointestinal tract ${ }^{15}$. In rare cases where a diagnosis of prostate cancer may change immediate management, we recommend that TRUS biopsies are performed using adequate $\mathrm{PPE}^{16}$ and strict adherence to appropriate antimicrobial prophylaxis. The risk of fecal transmission during a DRE is unknown and, to our knowledge, international societies have not addressed use of PPE during the examination. We recommend adherence to institutional Infection Prevention and Control (IPAC) guidelines. If unavailable, we recommend at minimum use of droplet precautions with mask, eye protection, gown and gloves.

\section{Treatment prioritization strategies}

Treatment recommendations depend on the predicted severity of disease, which we have defined below using National Comprehensive Cancer Network (NCCN) guidelines ${ }^{17}$.

\section{Localized low risk prostate cancer (very low, low and favourable intermediate risk (FIR) groups).}

General principle: In men with asymptomatic low risk prostate cancer, deferral of further investigations and treatments is recommended until return to routine clinical activities.

I. In patients currently on or choosing active surveillance short-term suspension of active surveillance protocols is recommended where appropriate, including in person clinic visits, DRE, PSA testing, imaging (including MRI) and repeat biopsy. 
II. In men choosing surgical treatment for low or FIR disease, delays of up to several months to one year from diagnosis to radical prostatectomy (RP) do not appear to worsen biochemical recurrence rates ${ }^{18-20}$. The length of delay until adverse outcomes occur is unknown, however 6-12 months is likely appropriate based on these retrospective series. Therefore, in men with newly diagnosed low risk prostate cancer (including FIR) consider delay of RP until return to routine elective procedures. Neoadjuvant androgen deprivation therapy (ADT) to bridge the COVID-19 related delay to RP should not be used in this patient population.

III. In men electing to proceed with radiation therapy (RT), a delay in treatment is also recommended. There is no role for neoadjuvant ADT in men with low risk prostate cancer, and it is not routinely used for FIR disease. Consultation with and referral to radiation oncology is advised where appropriate.

IV. In patients on ongoing surveillance following definitive therapy for low- and FIR disease consider decreasing frequency of PSA testing and deferring in-office clinic appointments, particularly for patients greater than 1 year since surgery or RT.

\section{Localized high risk prostate cancer (unfavorable intermediate risk (UIR), high risk (HR) and very high risk (VHR) groups)}

I. For new consults, we recommend proceeding with diagnostic interventions and staging investigations in these patients pending resource availability, since a finding of metastatic disease would significantly alter management.

II. Patients with UIR, HR and VHR prostate cancer who choose radiation treatment should begin neoadjuvant ADT (NADT), as per current best practice recommendations. Four to 6 months of NADT is appropriate for patients with UIR - note that RTOG 9910 showed that 9 months of ADT did not improve local control, biochemical disease-free survival, cancer-specific mortality, metastasisfree survival, or overall survival ${ }^{21}$. Hypofractionated RT protocols should be considered to minimize patient visits.

III. UIR, HR and VHR patients electing to proceed with radical prostatectomy require special consideration. Within the current COVID-19 climate many centers are deferring non-emergent surgical cases, therefore a delay in time to RP from diagnosis may be expected. In a retrospective analysis of UIR, HR and VHR patients, a treatment delay for up to 6 months did not affect BCR or recurrence free survival ${ }^{22}$, whereas a study of HR and VHR cases only suggested no adverse oncologic outcomes from a 3-month delay ${ }^{23}$. Hence, a delay of 3 months may be considered in places where surgical resource capacity is limited.

IV. Neoadjuvant ADT prior to RP for localized prostate cancer is not recommended outside of a clinical trial because current best available evidence suggests no overall survival benefit ${ }^{24}$. However, there is a significant improvement in multiple 
pathological variables, including nodal metastases and positive margins with an acceptable safety profile ${ }^{24}$. In a randomized study comparing 3- and 8-month durations of NADT prior to RP, patients in the 8-month group had ongoing pathological and biochemical regression of localized prostate cancer, suggesting safety of this approach ${ }^{25}$. Therefore, this option may be considered in patients with UIR, HR and VHR disease during the COVID-19 crisis if prolonged surgical delays are expected. Patients should be aware that this is not standard practice, and the risk-benefit discussion should be documented. Use of androgen receptor axis targeted therapies (ARAT) in this context remains experimental and is not recommended.

V. For patients on surveillance following definitive therapy for high risk prostate cancer we recommend ongoing PSA testing and imaging, if needed, to assess for recurrent disease. Consideration may be given to decreased frequency of testing in men who have been disease free for 2 years or greater, and to transition them to telehealth visits.

\section{Advanced prostate cancer (clinical nodal involvement, biochemical recurrence post primary treatment (BCR), metastatic disease)}

I. Patients with newly diagnosed advanced prostate cancer are complex and require comprehensive and preferably multi-disciplinary assessment. We recommend considering in-person clinic consultations for these men depending on clinical scenario. Full staging evaluation, including laboratory testing, and imaging is also recommended.

II. In men with high risk features post RP, early salvage RT is recommended over up front adjuvant $\mathrm{RT}^{26,27}$. Men with BCR and no evidence of metastases should have ongoing PSA and imaging assessments as indicated, and the frequency should be dictated by disease risk and PSA doubling time. Hypofractionated RT protocols should be considered to minimize patient visits.

III. Men with newly diagnosed node positive prostate cancer without evidence of further metastases should receive ADT and consideration for external beam RT as per current best practice. Hypofractionated RT protocols should be considered. Abiraterone has also shown benefit in these patients ${ }^{28}$, however this must be balanced with requirement for laboratory monitoring and physical examination. Therefore, we would recommend a delay of abiraterone therapy for of up to 6 months from time of diagnosis.

IV. In men with newly diagnosed metastatic hormone sensitive prostate cancer (HSPC), we recommend treatment with an ARAT over docetaxel chemotherapy in addition to ADT. While outcomes of prostate cancer patients infected with SARS-CoV-2 are unknown, cancer patients with a history of receiving 
chemotherapy within 1 month are at higher risk for severe illness ${ }^{2}$. Chemotherapy administration is also associated with more intense resource utilization and risk exposure.

V. Men with oligometastatic HSPC may benefit from ADT (with or without an ARAT) with external beam RT to the prostate ${ }^{29,30}$. We recommend withholding or delaying RT in this setting during the pandemic. If RT is administered, a hypofractionated course should be considered.

VI. In men with a new diagnosis of high risk (PSADT $<10$ months) nonmetastatic castrate resistant prostate cancer (nmCRPC) we recommend consideration of apalutamide, enzalutamide or darolutamide per current standard of care ${ }^{31-33}$. In nmCRPC patients with a prolonged PSADT we recommend considering a decrease in the frequency of imaging.

VII. In men with a new diagnosis of metastatic castrate resistant prostate cancer (mCRPC) who have not previously been treated with an ARAT, we recommend this therapy over chemotherapy for the reasons discussed above. Another option may be Radium-223 in men with bony metastases, however the benefit must be weiged against the risk of pancytopenia. Men should be referred to medical oncology for discussion of risks and benefits of systemic therapy within the COVID-19 setting.

VIII. In men with painful bone metastases or bone metastases at high risk of fracture (weight bearing bone such as vertebra/pelvis/femur) we recommend referral to radiation oncology for a short course of palliative radiotherapy.

\section{Special considerations}

1. The treatment of localized or locally advanced prostate cancer within the COVID-19 context requires complex decision making, not only with respect to timing but also choice of treatment modality. Surgery and brachytherapy carry the risk of serious complications, require utilization of hospital resources and have increased risk of SARS-CoV-2 exposure to patients and healthcare personnel. External beam RT mitigates some of these risks, however patients are subject to multiple, repeated outpatient hospital visits. Many RT groups have instituted short course interim policies leaning heavily on stereotactic body RT techniques ${ }^{34}$. The optimal choice and timing of treatment ultimately requires shared decision making and multidisciplinary collaboration.

2. For robotic-assisted laparoscopic prostatectomy or laparoscopic $\mathbf{R P}$ there may be an increased risk for aerosolization of the virus ${ }^{35}$. Although this has not yet been directly linked to SARS-CoV-2, urologists performing minimally invasive surgery should take necessary precautions to mitigate this possibility, including use of filter devices ${ }^{16,35}$. 
There are several filter devices available on the market, and they have been summarized elsewhere ${ }^{16}$.

3. For patients on ADT, strong consideration should be given to using longer acting depots and implementing home injection programs where available in order to decrease patient and healthcare practitioner exposures.

4. Special consideration should be given to patients on bone targeted therapies, specifically denosumab. For men with mCRPC receiving monthly dosing, self-injections should be encouraged when possible to limit exposure to healthcare personnel. The frequency of laboratory monitoring (calcium specifically) and associated exposure risk present an added challenge during the COVID-19 crisis. This must be balanced with the net benefit of therapy. In patients who are unable to or refusing laboratory testing during the pandemic, we recommend temporary discontinuation of denosumab, or consideration of longer treatment intervals (eg. 3 instead of 1 month).

5. For patients receiving abiraterone, the home monitoring program should be instituted to avoid unnecessary hospital and clinic visits.

6. For patients receiving and progressing on abiraterone, the switch from prednisone to dexamethasone is commonly practiced and may delay the time to initiation of chemotherapy $^{36}$. This may be advantageous in the setting of COVID-19. However, practitioners must be aware that there is currently a global shortage in access to dexamethasone.

7. Many institutions have restricted visitor access. This challenge may be of particular concern to patients with advanced prostate cancer, cognitive challenges or language barriers, whose caregivers are highly involved in treatment decisions and information synthesis. Patients should be encouraged to use technology (video, telephone) to enhance discussion and comprehension during the clinic visit.

8. Men with advanced prostate cancer are generally older, frail, and have multiple comorbidities in addition to an advanced malignancy. This makes them a vulnerable population during the COVID-19 pandemic. Patients and their families should be encouraged to discuss substitute decision making and advanced directives. A useful framework on this subject is discussed elsewhere ${ }^{37}$. 
9. One of the major repercussions of COVID-19 is the potential for economic instability and occupational insecurity. Many younger patients may not have continuing access to drug coverage benefits as a result. Use of compassionate drug access programs, if available, is strongly encouraged for these cases.

\section{Conclusions}

The COVID-19 pandemic has resulted in extraordinary challenges to healthcare systems, which raises several concerns for the treatment of prostate cancer patients. Herein we provide a framework for Canadian physicians managing this complex malignancy during a global health crisis, as summarized in Table 1. The proposed recommendations act as a guide and must be considered in the context of a fluctuating and evolving environment. They do not address the impact of potentially delayed care on the healthcare system once operations return to preCOVID-19 levels. We recognize that this is a complex issue and that delayed care may result in challenging triaging decisions in the future, however these recommendations are meant to guide physicians during the acute crisis phase. We note that population-wide changes to prostate cancer care are not unprecedented, with one population-based study showing a decrease in PSA detectable prostate cancer diagnoses and increased use of conservative management during the Great Recession ${ }^{38}$. We believe that the principles in this statement may remain applicable under future resource constraints.

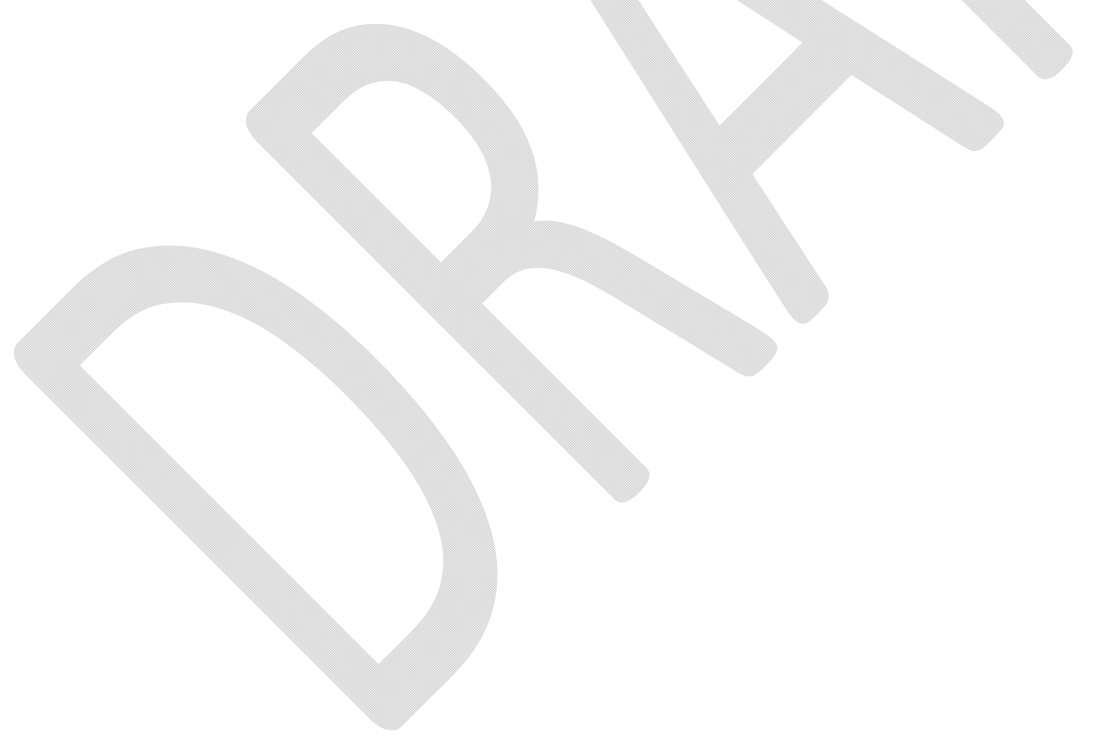




\section{References}

1. Bai Y, Yao L, Wei T et al. Presumed asymptomatic carrier transmission of COVID-19. JAMA 2020; 323:1406-7.

2. Liang W, Guan W, Chen $\mathrm{R}$ et al. Cancer patients in SARS-CoV-2 infection: a nationwide analysis in China. Lancet Oncol 2020; 21:335-7.

3. Desai A, Sachdeva S, Parekh T et al. COVID-19 and cancer: Lessons from a pooled meta-analysis. JCO Glob Oncol 2020; 6:557-9.

4. . Lei S, Jiang F, Su W et al. Clinical characteristics and outcomes of patients undergoing surgeries during the incubation period of COVID-19 infection. EClinicalMedicine 2020 [epub ahead of print]. doi: 10.1016/j.eclinm.2020.100331.

5. Buriki TK. Cancer guidelines during the COVID-19 pandemic. Lancet Oncol 2020 [epub ahead of print]. doi: 10.1016/S1470-2045(20)30217-5.

6. You B, Ravaud A, Canivete A et al. The official French guidelines to protect patients with cancer. Lancet Oncol 2020 [epub ahead of print]. doi: 10.1016/S14702045(20)30204-7.

7. Lalani AA, Chi KN, Heng DYC. Prioritizing systemic therapies for genitourinary malignancies: Canadian recommendations during the COVID-19 pandemic. Can Urol Assoc J 2020. [epub ahead of print]. doi: 10.5489/cuaj.6595.

8. NCCN, Care of prostate cancer patients during the COVID-19 pandemic: Recommendations of the NCCN. [Online, Accessed April 16, 2020] https://www.nccn.org/covid-19/pdf/NCCN_PCa_COVID_guidelines.pdf.

9. Ferreira R, McGrath M, Wang $\mathrm{Y}$ et al. How to prioritize urologic surgeries during epidemics: Lessons learned from the Toronto SARS outbreak in 2003. Can Urol Assoc J 2020. [epub ahead of print]. https://doi.org/10.5489/cuaj.6551

10. Wilt TJ, Jones KM, Barry MJ et al. Follow-up of prostatectomy versus observation for early prostate cancer. $N$ Engl J Med 2017; 377:132-42.

11. Hamdy FC, Donovan JL, Lane JA et al. 10-Year outcomes after monitoring, surgery, or radiotherapy for localized prostate cancer. $N$ Engl J Med 2016; 375: 1415-24.

12. Ravi P, Karakiewicz PI, Roghmann F. Mental health outcomes in elderly men with prostate cancer. Urol Oncol 2014; 32: 1333-40.

13. Watts S, Leydon G, Birch B et al. Depression and anxiety in prostate cancer: a systematic review and meta-analysis of prevalence rates. BMJ Open 2014; 4: e003901.

14. Rendon RA, Mason RJ, Marzouk K et al. Canadian Urological Association recommendations on prostate cancer screening and early diagnosis. Can Urol Assoc $J$ 2017; 11: 298-309.

15. Hindson J. COVID-19: faecal-oral transmission? Nat Rev Gastroenterol Hepatol 2020. [epub ahead of print]. doi: 10.1038/s41575-020-0295-7.

16. SAGES and EAES Recommendations Regarding Surgical Response to COVID-19 Crisis. [Online] [Accessed April 16, 2020 ] https://www.sages.org/recommendations-surgicalresponse-covid-19/.

17. National Comprehensive Cancer Network. Prostate Cancer (Version 1.2020) [Online] [Accessed April 16, 2020]

https://www.nccn.org/professionals/physician_gls/pdf/prostate.pdf. 
18. Khan MA, Mangold LA, Epstein JI et al. Impact of surgical delay on long-term cancer control for clinically localized prostate cancer. J Urol 2004; 172: 1835-9.

19. Freedland SJ, Kane CJ, Amling CL et al. Delay of radical prostatectomy and risk of biochemical progression in men with low risk prostate cancer. J Urol 2006; 175: 1298302.

20. Boorjian S, Bianco F, Scardino $\mathrm{P} t$ al. Does the time from biopsy to surgery affect biochemical recurrence after radical prostatectomy? BJU Int 2005; 96: 773-76.

21. Pisansky TM, Hunt D, Gomella LG et al. Duration of androgen suppression before radiotherapy for localized prostate cancer: Radiation therapy oncology group randomized Clinical Trial 9910 . J Clin Oncol 2015; 33: 332-9.

22. Gupta N, Bivalacqua TJ, Han M et al. Evaluating the length of time from diagnosis to surgery in patients with unfavourable intermediate risk to very-high risk clinically localised prostate cancer. BJU Int 2019; 124: 268-74 .

23. Reichard CA, Nyame YA, Sundi D et al. Does time from diagnosis to treatment of highor very-high-risk prostate cancer affect outcome? BJU Int 2019; 124: 282-9.

24. Kumar S, Shelley M, Harrison $\mathrm{C}$ et al. Neo-adjuvant and adjuvant hormone therapy for localised and locally advanced prostate cancer. Cochrane Database Sys Rev 2006; Art. No.: CD006019.

25. Gleave ME, Goldenberg SL, Chin JL et al. Randomized comparative study of 3 versus 8month neoadjuvant hormonal therapy before radical prostatectomy: biochemical and pathological effects. J Urol 2001; 166: 500-7.

26. Parker C, Clarke NW, Cook A et al. LBA49_PR - Timing of radiotherapy (RT) after radical prostatectomy (RP): First results from the RADICALS RT randomised controlled trial (RCT) [NCT00541047]. Ann Oncol 2019; 30 (Suppl 5): v883-4.

27. Vale CL, Brihoum M, Chabaud S et al. Adjuvant or salvage radiotherapy for the treatment of localised prostate cancer? A prospectively planned aggregate data metaanalysis. Ann Oncol 2019; 30 (Suppl 5): v883.

28. James ND, de Bono JS, Spears MR et al. Abiraterone for prostate cancer not previously treated with hormone therapy. N Engl J Med 2017; 377: 338-51.

29. Parker CC, James ND, Brawley CD et al. Radiotherapy to the primary tumor for newly diagnosed, metastatic prostate cancer (STAMPEDE): a randomised controlled phase 3 trial. Lancet 2018; 392: 2353-66.

30. Boevé LMS, Hulshof MCCM, Vis AN et al. Effect on survival of androgen deprivation therapy alone compared to androgen deprivation therapy combined with concurrent radiation therapy to the prostate in patients with primary bone metastatic prostate cancer in a prospective randomised clinical trial. Eur Urol 2019; 75: 410-18.

31. Hussain M, Fizazi K, Saad F et al. Enzalutamide in men with nonmetastatic, castrate resistant prostate cancer. $N$ Engl J Med 2018; 378: 2465-74.

32. Smith MR, Saad F, Chowdhury S et al. Apalutamide treatment and metastasis-free survival in prostate cancer. $N$ Engl J Med 2018; 378: 1408-18.

33. Fizazi K, Shore N, Tammela TL et al. Darolutamide in nonmetastatic, castrate-resistant prostate cancer. $N$ Engl J Med 2019; 380: 1235-46. 
34. Simcock R, Thomas TV, Mercy CE et al. COVID-19: Global radiation oncology's targeted response for pandemic preparedness. Clin Transl Rad Onc 2020. [epub ahead of print]. doi: https://doi.org/10.1016/j.ctro.2020.03.009.

35. Zheng MH, Boni L, Fingerhut A et al. Minimally invasive surgery and the novel coronavirus outbreak: Lessons from China and Italy. Ann Surg 2020. [epub ahead of print]. DOI: 10.1097/SLA.0000000000003924.

36. Romero-Laorden N, Lozano R, Jayaram A et al. Phase II pilot study of the prednisone to dexamethasone switch in metastatic castration-resistant prostate cancer (mCRPC) patients with limited progression on abiraterone plus prednisone (SWITCH study). Br J Cancer 2018; 119: 1052-59.

37. Curtis JR. The importance of addressing advance care planning and decisions about donot-resuscitate orders during the novel coronavirus-2019 (COVID-19). JAMA 2020. [epub ahead of print]. doi: 10.1001/jama.2020.4894.

38. Weiner AB, Conti RM, Eggener SE. National economic conditions and patient insurance status predict prostate cancer diagnosis rates and management decisions. J Urol 2016; 195: 1383-89.

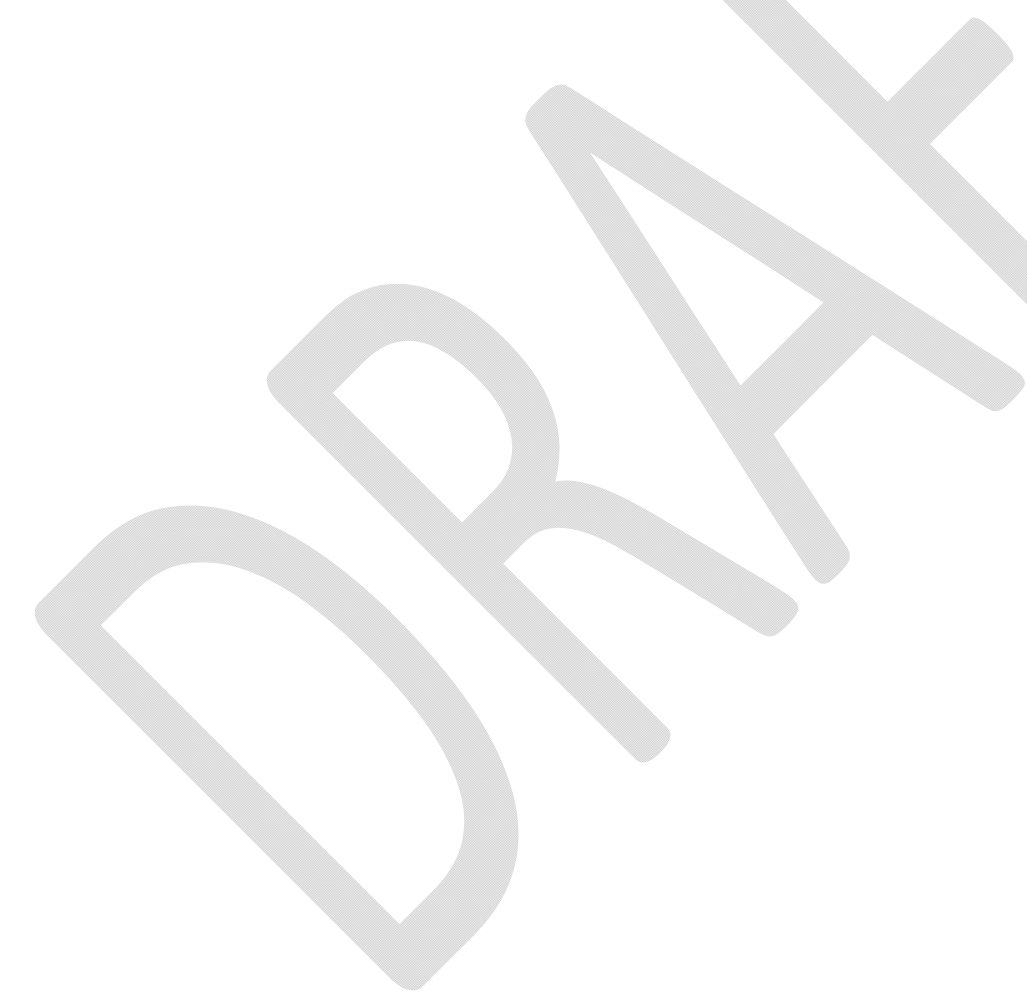


Figures and Tables

\begin{tabular}{|l|l|l|l|l|}
\hline Table 1. Summary of treatment recommendations for prostate cancer during COVID-19 pandemic \\
\hline & In-person consult & $\begin{array}{l}\text { Diagnostic } \\
\text { investigations } \\
\text { (imaging, biopsy) }\end{array}$ & Treatment & Post-treatment monitoring \\
\hline $\begin{array}{l}\text { Very low-risk } \\
\text { Low-risk } \\
\text { Favourable } \\
\text { intermediate-risk }\end{array}$ & Not recommended & Not recommended & $\begin{array}{l}\text { Recommend delaying until pandemic } \\
\text { resolution }\end{array}$ & $\begin{array}{l}\text { Recommend delaying active } \\
\text { surveillance protocols }\end{array}$ \\
\hline $\begin{array}{l}\text { Unfavourable } \\
\text { intermediate-risk } \\
\text { High-risk } \\
\text { Very high-risk } \\
\text { depending on } \\
\text { clinical scenario }\end{array}$ & $\begin{array}{l}\text { May be considered, } \\
\text { Recommended }\end{array}$ & $\begin{array}{l}\text { Cooadjuvant ADT for patients } \\
\text { Choosing RT } \\
\text { if extended delay to surgery } \\
\text { Consider delaying definitive therapy } \\
\text { (up to 3 months from diagnosis) }\end{array}$ & $\begin{array}{l}\text { Recommend ongoing } \\
\text { surveillance } \\
\text { Consider decreasing } \\
\text { frequency of PSA and } \\
\text { imaging if }>2 \text { years since } \\
\text { definitive therapy and stable }\end{array}$ \\
\hline $\begin{array}{l}\text { Locally advanced } \\
\text { or metastatic }\end{array}$ & $\begin{array}{l}\text { May be considered, } \\
\text { depending on } \\
\text { clinical scenario }\end{array}$ & Recommended & $\begin{array}{l}\text { Recommended (see text for details) } \\
\text { Rentients on treatment is } \\
\text { recommended to monitor for } \\
\text { disease progression and AEs }\end{array}$ \\
\hline
\end{tabular}

ADT: androgen deprivation therapy; AE: adverse events; PSA: prostate-specific antigen; RP: radical prostatectomy; RT: radiotherapy. 